Résumés des conférences et travaux

\title{
Claude Nicolet (1930-2010)
}

Jean-Louis Ferrary

\section{OpenEdition \\ Journals}

Édition électronique

URL : https://journals.openedition.org/ashp/1359

DOI : 10.4000/ashp.1359

ISSN : 1969-6310

Éditeur

Publications de l'École Pratique des Hautes Études

\section{Édition imprimée}

Date de publication : 1 octobre 2012

Pagination : xxi-xxiii

ISSN : 0766-0677

\section{Référence électronique}

Jean-Louis Ferrary, "Claude Nicolet (1930-2010) », Annuaire de l'École pratique des hautes études (EPHE), Section des sciences historiques et philologiques [En ligne], 143|2012, mis en ligne le 27 septembre 2012, consulté le 03 août 2021. URL : http://journals.openedition.org/ashp/1359 ; DOI : https://doi.org/10.4000/ashp.1359 


\section{CLAUDE NICOLET \\ (1930-2010)}

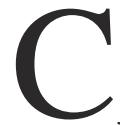

LAUde Nicolet est mort à Paris le 24 décembre 2010. Il était né à Marseille le 15 septembre 1930, et c'est dans cette ville qu'il avait fait toutes ses études, jusqu'à l'entrée à l'École normale supérieure de la rue d'Ulm en 1950. Agrégé d'histoire en 1954, il fut pendant deux ans professeur au lycée d'Avignon, mais en 1956 il entra au Cabinet de Pierre Mendès France, alors ministre d'État sans portefeuille dans le gouvernement de Guy Mollet. Brève expérience, puisque Mendès France, en désaccord avec la politique algérienne de Guy Mollet, allait démissionner dès le mois de mai. Mais l'engagement de Claude Nicolet dans le mendésisme allait continuer à la tête des Cahiers de la République (1956-1963), et ses deux premiers livres furent un «Que sais-je? » sur Le radicalisme, publié en 1957, et l'essai sur Pierre Mendès France ou le métier de Cassandre, de 1959. Toute sa vie, Claude Nicolet garda, non seulement un intérêt profond pour la réflexion politique, mais une attirance pour une carrière politique, qui le conduisit à une candidature infructueuse à la députation en 1973, puis une sorte de regret face à ce qu'il considérait comme un inaccomplissement. Du moins devait-il trouver en Jean-Pierre Chevènement un homme politique attentif à ses idées, qui lui confia à deux reprises une mission de réflexion sur l'instruction civique, en tant que ministre de l'Éducation nationale en 1984-1985, puis en tant que ministre de la défense en 1989-1991.

En attendant, Claude Nicolet devenait membre de l'École française de Rome (1957-1959), puis maître de conférences à l'université de Tunis en 1959, jusqu'à ce qu'en 1961 une prise de position publique sur la crise de Bizerte fût sanctionnée par son rappel en France, et sa rétrogradation comme maître-assistant à l'université de Caen. Depuis l'École normale, il avait travaillé sous la direction de William Seston, mais s'était résolument orienté vers l'histoire de la Rome républicaine. C'est en 1966 qu'il devint docteur ès lettres, avec une thèse principale sur L'Ordre équestre à l'époque républicaine, et comme thèse secondaire une Prosopographie des chevaliers romains qui parut en 1974. Contre la théorie alors courante de l'ordre équestre comme « middle class », il imposa l'idée que sénateurs et chevaliers appartenaient en fait à une même classe sociale, mais constituaient deux ordres bien distincts, dont chacun jouait son rôle dans la cité, surtout à partir des Gracques, lorsque l'ordre équestre disputa la judicature aux sénateurs et qu'il contrôla les grandes sociétés de publicains. Indissociable de la thèse est l'organisation du colloque « Groupes sociaux, ordres et classes dans l'Antiquité gréco-romaine » qui se tint à Caen en avril 1969 et qui fut publié en 1970 sous le titre Recherches sur les structures sociales dans l'Antiquité classique et, la même année 1970, l'article des Annales sur " Prosopographie et histoire sociale ». Entre temps, en 1969, Claude Nicolet avait simultanément été élu professeur d'histoire romaine à la Sorbonne encore unifiée (il choisira ensuite de rejoindre Paris-I), et directeur d'études dans notre section, avec une conférence sur les « Institutions politiques de Rome ». 
La conférence de l'EPHE, qui se déroulait le mardi de 16 à 18 heures et était suivi de longues conversations dans l'un ou l'autre des cafés proches de la Sorbonne, devint rapidement l'un des centres majeurs de la recherche en histoire romaine. On n'y trouvait pas seulement les élèves de Nicolet, dont beaucoup allaient à leur tour devenir des maîtres (Élizabeth Deniaux, François Hinard, Mireille Cébeillac, Ségolène Demougin, Jean-Michel David, Jean-Marc Flambard, Marianne Coudry, Catherine Virlouvet, Claudia Moatti, Pierre Côme, Yann Rivière), mais bien d'autres jeunes historiens ainsi que des latinistes dont certains se sont mués en historiens (John Scheid dès la première année; Jean Andreau, Jean Christian Dumont, Philippe Moreau et moi-même, qui préparions nos thèses sous la direction de Pierre Grimal; Mouza Raskolnikoff, mais aussi Mireille Corbier, Patrick Le Roux, Agnès Rouveret, Sylvie Pittia), des juristes enfin (Yan Thomas, Jean-Pierre Coriat). Les étrangers aussi étaient nombreux (Élie Fallu et Hélène Leclerc, du Canada, Ella Hermon, Benjamin Cohen et Jacob Stern, d'Israël, Paula Botteri, d'Italie, Michel Dubuisson et Étienne Famerie, de Belgique). Cette liste n'est pas exhaustive, bien sûr. Le plus remarquable est la fidélité avec laquelle les plus anciens de ses auditeurs revenaient régulièrement. Les plus jeunes étaient sans doute impressionnés par leur présence autour de la grande table, alors qu'ils se contentaient le plus souvent des chaises du deuxième rang, mais après avoir passé l'épreuve d'une ou deux questions insidieuses du maître, ils s'habituaient vite, et s'intégraient au groupe. La conférence de l'EPHE fut véritablement l'atelier où Claude Nicolet construisit l'essentiel de son œuvre, soumise aux critiques d'un public de collègues plus jeunes, pour qui le respect n'impliquait aucune inhibition, et qui n'hésitaient pas à soulever des objections, parfois infondées, mais d'autres fois méritées, et alors prises en compte par le maître. La fiscalité est une sorte de fil rouge qui apparaît dès les premières années et revient régulièrement, à l'occasion de documents nouveaux comme le règlement de la douane de la province d'Asie, même si la synthèse longtemps attendue sur les sociétés de publicains ne parut jamais. Un autre thème majeur des premières années fut la prosopographie, qui ne disparut jamais totalement mais s'estompa, les grands dossiers étant devenus les thèses des premiers disciples. La perspective s'élargit avec les années de rédaction du Métier de citoyen (1976) et des Structures de l'Italie romaine (1977), puis du gros chapitre sur la pensée économique des Romains dans la Storia delle idee politiche, economiche e sociali de Luigi Firpò (1982), mais la grande mutation fut la perspective impériale, au sens géographique mais aussi chronologique, qui allait donner L'inventaire du monde. Géographie et politique aux origines de l'Empire romain (1988), et d'une certaine façon se prolonger dans la grande enquête sur La Mémoire perdue. Recherches sur les archives publiques et privées dans la Rome antique (1993). La conférence fut aussi un exemple remarquable d'incitation à la recherche pour certains des auditeurs, qui furent étroitement associés à plusieurs publications collectives : « Le Commentariolum petitionis de Quintus Cicéron. État de la question et étude prosopographique » (1972) n'était encore qu'un gros article, mais suivirent les volumes sur Insula sacra. La loi Gabinia Calpurnia de Délos (1980), Demokratia et aristokratia. À propos de C. Gracchus : mots grecs et réalités romaines (1983), Des ordres à Rome (1984), ouvrages directement liés à des recherches conduites dans le cadre de la conférence. S'il n'aimait pas trop faire parler d'autres que lui pendant toute une conférence, car il avait l'impression, me disait-il, de voler alors l'argent de la République, si, à de rares exceptions, il réservait ce privilège à d'illustres étrangers de passage par Paris, 
de Sir Ronald Syme à Filippo Coarelli, Claude Nicolet, en revanche, aimait faire participer ses disciples à la recherche menée à l'École. Les ouvrages collectifs n'en sont pas la seule preuve : avec une extraordinaire générosité, lorsqu'il n'avait pas le temps de poursuivre une piste et que de nouveaux sujets, de nouvelles découvertes l'attiraient, il n'hésitait jamais à confier à l'un de ses disciples qui s'était montré particulièrement intéressé le soin de continuer la recherche, en se contentant de lui confier ses notes et de lui prodiguer ses conseils. C'est ainsi que bien des articles, et parfois des livres, où le nom de Claude Nicolet n'apparaît que dans des lignes de remerciement, devraient être ajoutés à la bibliographie issue du séminaire. Ajoutons encore que, pour bien des auditeurs (et c'est mon cas), la conférence de Claude Nicolet fut l'occasion de découvrir aussi l'intérêt des études historiographiques. Il est tout un pan de son œuvre qui fut totalement écrit en marge de son enseignement à l'École, en particulier son grand livre sur L'idée républicaine en France de 1982. Mais nous eûmes à partir de 1979/1980 les premières réflexions, l'écho des premières recherches qui allaient être la matière de son dernier livre : La fabrique d'une nation. La France entre Rome et les Germains (2003).

Cette grande aventure, qui marqua tous ceux qui y participèrent, prit fin lorsque Claude Nicolet fut nommé à la tête de l'École française de Rome, qu'il dirigea de 1992 à 1995. On ne regrettera pas bien sûr l'action administrative et scientifique qu'il put mener dans cette institution qui lui était si chère, et dont l'un des fruits fut le gros et beau volume sur Les mégapoles méditerranéennes (1998). Mais lorsqu'une application stricte de la « loi Desgraupes » écourta son mandat et qu'il dut revenir à Paris, et même s'il ne prit sa retraite qu'en 1998, l'élan n'était plus le même, et la conférence ne retrouva pas son éclat et son extraordinaire dynamisme d'avant Rome.

Claude Nicolet eut la reconnaissance nationale et internationale due au très grand savant qu'il était. Élu en 1986 à l'Académie des inscriptions et belles lettres, il devint aussi membre de la British Academy et de l'Accademia dei Lincei. Il fut fait docteur honoris causa de l'université de Liège et de l'université libre de Bruxelles. Il donna des " Jerome Lectures ", à Ann Arbor et à Rome, et avait été invité à donner aussi à Berkeley des « Sather Lectures », même s'il dut décliner cette proposition. En Italie, il reçut en 1994 le prestigieux « Premio Galileo Galilei », et en 1997 le « Premio Cultore di Roma ». Après la parution de Fabrique d'une nation, son activité se ralentit, et son dernier travail aura été l'hommage qu'il devait à son maître : la « Notice sur la vie et les travaux de Wiliam Seston » lue dans la séance de l'Académie du 19 octobre 2007. C'est pendant l'été 2009 que se révéla la gravité du mal qui devait l'emporter. Je n'ai pas eu aujourd'hui la prétention de dresser un tableau de la totalité son œuvre et, comme il se devait, j'ai surtout mis l'accent sur l'importance de sa conférence des Hautes Études, qui a tant marqué ceux qui en ont été les auditeurs. À l'homme généreux et aimé, au savant, qui fut l'un des plus grands spécialistes de la Rome antique mais ne cessa pas non plus de réfléchir sur la République et la nation françaises, un hommage a été rendu le 28 mai 2011 à l'INHA, à l'initiative de plusieurs de ses disciples, dont Ségolène Demougin et moi-même, et sous le patronage des établissements et institutions où son activité s'est déployée, parmi lesquels, bien entendu, l'École pratique des hautes études. Les actes en paraîtront bientôt, avec une bibliographie de ses travaux, dans le no 22 (2011) des Cahiers du Centre Gustave Glotz. 